\title{
An Almost Sure Approximation for the Predictable Process in the Doob-Meyer Decomposition Theorem
}

\author{
Adam Jakubowski ${ }^{\star}$ \\ Nicolaus Copernicus University, \\ Faculty of Mathematics and Computer Science, \\ ul. Chopina 12/18, 87-100 Torun, Poland. \\ e-mail: adjakubo@mat.uni.torun.pl
}

Summary. We construct the Doob-Meyer decomposition of a submartingale as a pointwise superior limit of decompositions of discrete submartingales suitably built upon discretizations of the original process. This gives, in particular, a direct proof of predictability of the increasing process in the Doob-Meyer decomposition.

\section{The Doob-Meyer Theorem}

The Doob-Meyer decomposition theorem opened the way towards the theory of stochastic integration with respect to square integrable martingales and - consequently - semimartingales, as described in the seminal paper [7]. According to Kallenberg [4], this theorem is "the cornerstone of the modern probability theory". It is therefore not surprising that many proofs of it are known. To the author's knowledge, all the proofs heavily depend on a result due to Doléans-Dade [3], which identifies predictable increasing processes with "natural" increasing processes, as defined by Meyer [6].

In the present paper we develop ideas of another classical paper by $\mathrm{K}$. Murali Rao [8] and construct a sequence of decompositions for which the superior limit is pointwise (in $(t, \omega)$ ) equal to the desired one, and thus we obtain predictability in the easiest possible way.

Let $\left(\Omega, \mathcal{F},\left\{\mathcal{F}_{t}\right\}_{t \in[0, T]}, P\right)$ be a stochastic basis, satisfying the "usual" conditions, i.e. the filtration $\left\{\mathcal{F}_{t}\right\}$ is right-continuous and $\mathcal{F}_{0}$ contains all $P$-null sets of $\mathcal{F}_{T}$. Let $(\mathrm{D})$ denote the class of measurable processes $\left\{X_{t}\right\}_{t \in[0, T]}$ such that the family $\left\{X_{\tau}\right\}$ is uniformly integrable, where $\tau$ runs over all stopping times with respect to $\left\{\mathcal{F}_{t}\right\}_{t \in[0, T]}$. One of the variants of the Doob-Meyer theorem can be formulated as follows.

\footnotetext{
* Supported by Komitet Badań Naukowych under Grant No PB 0253/P03/2000/19 and completed while the author was visiting Université de Rouen. The author would like to thank people of Mathematics in Rouen for their hospitality.
} 
Theorem 1. Any submartingale of class (D) admits a unique decomposition $X_{t}=M_{t}+A_{t}$, where $\left\{M_{t}\right\}$ is a uniformly integrable martingale and $\left\{A_{t}\right\}$ is a predictable increasing process, with $A_{T}$ integrable.

In discrete time this theorem is trivial: if $\left\{X_{k}\right\}_{k=0,1, \ldots, k_{0}}$ is a submartingale with respect to $\left\{\mathcal{F}_{k}\right\}_{k=0,1, \ldots, k_{0}}$, we can set $A_{0}=0$ and

$$
A_{k}=\sum_{j=1}^{k} E\left(X_{j}-X_{j-1} \mid \mathcal{F}_{j-1}\right), \quad k=1,2, \ldots, k_{0} .
$$

The appealing idea of Murali Rao [8] consists in approximating $A_{t}$ by increasing processes defined by discretizations of $X_{t}$.

Let $\theta_{n}=\left\{0=t_{0}^{n}<t_{1}^{n}<t_{2}^{n}<\ldots<t_{k_{n}}^{n}=T\right\}, n=1,2, \ldots$, be an increasing sequence of partitions of $[0, T]$, with

$$
\max _{1 \leqslant k \leqslant k_{n}} t_{k}^{n}-t_{k-1}^{n} \longrightarrow 0, \quad \text { as } n \rightarrow \infty .
$$

By "discretizations" $\left\{X_{t}^{n}\right\}_{t \in \theta_{n}}$ of $\left\{X_{t}\right\}_{t \in[0, T]}$ we mean the processes defined by

$$
X_{t}^{n}=X_{t_{k}^{n}} \quad \text { if } \quad t_{k}^{n} \leqslant t<t_{k+1}^{n}, X_{T}^{n}=X_{T} .
$$

The process $X^{n}$ is a submartingale with respect to the discrete filtration $\left\{\mathcal{F}_{t}\right\}_{t \in \theta_{n}}$ and by the above discrete scheme we obtain a sequence of right continuous representations

$$
X_{t}^{n}=M_{t}^{n}+A_{t}^{n}
$$

where

$$
\begin{aligned}
& A_{t}^{n}=0 \quad \text { if } \quad 0 \leqslant t<t_{1}^{n}, \\
& A_{t}^{n}=\sum_{j=1}^{k} E\left(X_{t_{j}^{n}}-X_{t_{j-1}^{n}} \mid \mathcal{F}_{t_{j-1}^{n}}\right) \quad \text { if } \quad t_{k}^{n} \leqslant t<t_{k+1}^{n}, k=1,2, \ldots, k_{n}-1, \\
& A_{T}^{n}=\sum_{j=1}^{k_{n}} E\left(X_{t_{j}^{n}}-X_{t_{j-1}^{n}} \mid \mathcal{F}_{t_{j-1}^{n}}\right) .
\end{aligned}
$$

Since $A_{t}^{n}$ is $\mathcal{F}_{t_{k-1}^{n}}$-measurable for $t_{k}^{n} \leqslant t<t_{k+1}^{n}$, the processes $A^{n}$ are predictable in the very intuitive manner.

The following facts can be extracted from [8].

Theorem 2. If $\left\{A_{t}\right\}$ is continuous (equivalently: $\left\{X_{t}\right\}$ is "quasi-left continuous", or "regular" in the former terminology), then for $t \in \bigcup_{n=1}^{\infty} \theta_{n}$

$$
A_{t}^{n} \longrightarrow A_{t} \quad \text { in } L^{1}
$$

In the general case

$$
A_{t}^{n} \longrightarrow A_{t} \quad \text { weakly in } L^{1}, \quad t \in \bigcup_{n=1}^{\infty} \theta_{n} .
$$


The latter statement cannot be improved: by a counterexample due to Dellacherie and Doléans-Dade [2], there exists an increasing integrable process $\left\{X_{t}\right\}$ and a sequence $\theta_{n}$ of partitions of $[0,1]$ such that the $A_{1}^{n}$ 's fail to converge in $L^{1}$ to $A_{1}$.

By a slight modification of the approximating sequence we can obtain convergence in the strong sense.

Theorem 3. There exists a subsequence $\left\{n_{j}\right\}$ such that for $t \in \bigcup_{n=1}^{\infty} \theta_{n}$ and as $J \rightarrow+\infty$

$$
\frac{1}{J}\left(\sum_{j=1}^{J} A_{t}^{n_{j}}\right) \longrightarrow A_{t}, \quad \text { a.s. and in } L^{1} .
$$

Remark 1 .

1. In fact, in any subsequence we can find a further "good" subsequence with the property described in Theorem 3. In view of Komlós' Theorem 4 below, it is natural to say that the sequence $\left\{A^{n}\right\}$ is $K$-convergent to $A$.

2. We do not know whether the whole sequence converges in the Cesàro sense.

\section{Proof of Theorem 3}

In order to avoid repetitions of well-known computations, we choose the textbook [4] as a fixed reference and will refer to particular results therein.

The preparating steps are standard and are given on pages 413-4 in [4].

1. If $X$ is a submartingale of class (D), then the family of all random variables of the form $\left\{A_{\tau_{n}}^{n}\right\}$, where $\tau_{n}$ is a stopping time taking values in $\theta_{n}$, is uniformly integrable. In particular,

$$
\sup _{n} E A_{T}^{n}<+\infty .
$$

2. We can extract a subsequence $\left\{n_{k}\right\}$ such that $A_{T}^{n_{k}} \rightarrow \alpha$ weakly in $L_{1}$. We define

$$
M_{t}=E\left(X_{T}-\alpha \mid \mathcal{F}_{t}\right), \quad A_{t}=X_{t}-M_{t} .
$$

Then we have also

$$
A^{n_{k}}(t) \longrightarrow A_{t} \quad \text { weakly in } L^{1}, \quad t \in \bigcup_{n=1}^{\infty} \theta_{n} .
$$

In the main step of proof we use the famous theorem of Komlós [5] (see also [1] for the contemporary presentation related to exchangeability). 
Theorem 4. If $\xi_{1}, \xi_{2}, \ldots$ is a sequence of random variables for which

$$
\sup _{n} E\left|\xi_{n}\right|<+\infty
$$

then there exists a subsequence $\left\{n_{j}\right\}$ and an integrable random variable $\xi_{\infty}$ such that for every subsequence $\left\{n_{j_{k}}\right\}$ of $\left\{n_{j}\right\}$ we have with probability one, as $K \rightarrow+\infty$,

$$
\frac{\xi_{n_{j_{1}}}+\xi_{n_{j_{2}}}+\cdots+\xi_{n_{j_{K}}}}{K} \longrightarrow \xi_{\infty} .
$$

By this theorem we can find a subsequence $\left\{n_{k_{j}}\right\} \subset\left\{n_{k}\right\}$ and a random variable $\alpha_{T}$ such that

$$
\frac{1}{J}\left(\sum_{j=1}^{J} A_{T}^{n_{k_{j}}}\right) \longrightarrow \alpha_{T}, \quad \text { a.s. }
$$

Since $A_{T}^{n_{k}} \rightarrow \alpha=A_{T}$ weakly in $L^{1}$, the Cesàro means of any subsequence also converge weakly to the same limit and we have $\alpha_{T}=A_{T}$. Since the family $\left\{A_{T}^{n}\right\}$ is uniformly integrable, the above convergence holds in $L^{1}$ as well.

Now let us take any $t_{0} \in \bigcup_{n=1}^{\infty} \theta_{n}, t_{0} \neq T$. As before, one can find another subsequence $\left\{n_{k_{j_{i}}}\right\} \subset\left\{n_{k_{j}}\right\}$ such that

$$
\frac{1}{I}\left(\sum_{i=1}^{I} A_{t_{0}}^{n_{k_{j_{i}}}}\right) \longrightarrow A_{t_{0}}, \quad \text { a.s. and in } L^{1} \text {. }
$$

By the exceptional "subsequence property" given in the Komlós theorem we can still claim that

$$
\frac{1}{I}\left(\sum_{i=1}^{I} A_{T}^{n_{k_{j_{i}}}}\right) \longrightarrow A_{T}, \quad \text { a.s. and in } L^{1} .
$$

Repeating these steps for each $t_{0} \in \bigcup_{n=1}^{\infty} \theta_{n}$ and then applying the diagonal procedure we find a subsequence fulfilling the requirements of Theorem 3.

It remains to identify the limit with the unique predictable increasing process given by the Doob-Meyer decomposition. This can be done using Rao's result, but given almost sure convergence everything can be done with bare hands:

\section{Predictability_-direct!}

We shall provide a direct proof of predictability of the process $A$ appearing as the limit in Theorem 3. For notational convenience we assume that (1) holds for the whole sequence $A^{n}$. We introduce two auxiliary sequences of stochastic processes given by the following formula. 


$$
\begin{aligned}
\tilde{A}_{0}^{n} & =0 \\
\tilde{A}_{t}^{n} & =\sum_{j=1}^{k} E\left(X_{t_{j}^{n}}-X_{t_{j-1}^{n}} \mid \mathcal{F}_{t_{j-1}^{n}}\right) \quad \text { if } \quad t_{k-1}^{n}<t \leqslant t_{k}^{n}, k=1,2, \ldots, k_{n}, \\
\tilde{B}_{t}^{n} & =\frac{1}{n} \sum_{j=1}^{n} \tilde{A}_{t}^{j} .
\end{aligned}
$$

The processes $\tilde{A}^{n}$ are adapted to the filtration $\left\{\mathcal{F}_{t}\right\}_{t \in[0, T]}$ and their trajectories are left continuous, hence they are predictable by the very definition of the predictable $\sigma$-field. The same holds for the $\tilde{B}^{n}$.

It is sufficient to show that there exists a set $E$ of probability zero such that for every $\omega \notin E$ and every $t \in[0, T]$

$$
\limsup _{n \rightarrow \infty} \tilde{B}_{t}^{n}(\omega)=A_{t}(\omega) .
$$

We have for $t_{0} \in \bigcup_{n=1}^{\infty} \theta_{n}$ and $n$ large enough

$$
\tilde{A}_{t_{0}}^{n}(\omega)=A_{t_{0}}^{n}(\omega)
$$

hence outside of a set $E^{\prime}$ of probability zero

$$
\tilde{B}_{t_{0}}^{n}(\omega) \rightarrow A_{t_{0}}(\omega) .
$$

Since $\bigcup_{n=1}^{\infty} \theta_{n}$ is dense in $[0, T]$, it follows that for $\omega \notin E^{\prime}$, in every point of continuity of $A_{(\cdot)}(\omega)$ we have

$$
\tilde{B}_{t}^{n}(\omega) \longrightarrow A_{t}(\omega)
$$

Moreover, since $A$ is right continuous we have always

$$
\limsup _{n \rightarrow \infty} \tilde{B}_{t}^{n}(\omega) \leqslant A_{t}(\omega)
$$

We conclude that (2) can be violated only in points of discontinuity of $A$.

We claim it suffices to prove that for each stopping time $\tau$

$$
\lim _{n \rightarrow \infty} E \tilde{A}_{\tau}^{n} \longrightarrow E A_{\tau} .
$$

To see this let us observe that if (3) holds then

$$
E \limsup _{n \rightarrow \infty} \tilde{B}_{\tau}^{n} \leqslant E A_{\tau}=\lim _{n \rightarrow \infty} E \tilde{B}_{\tau}^{n} \leqslant E \limsup _{n \rightarrow \infty} \tilde{B}_{\tau}^{n},
$$

where Fatou's lemma can be applied in the last inequality because

$$
\tilde{B}_{\tau}^{n} \leqslant B_{T}^{n} \longrightarrow A_{T} \quad \text { in } L^{1} .
$$

In particular, for every stopping time $\tau$, we have almost surely, 


$$
A_{\tau}=\limsup _{n \rightarrow \infty} \tilde{B}_{\tau}^{n}
$$

Now it is well known (and easy to prove in the case of increasing processes) that there exists a sequence $\left\{\tau_{q}\right\}$ of stopping times which exhaust all jumps of $A$, i.e. $P\left(\Delta A_{\tau}>0\right)>0$ implies $P\left(\tau=\tau_{q}\right)>0$ for some $q$. For each $q$ we have

$$
A_{\tau_{q}}=\limsup _{n \rightarrow \infty} \tilde{B}_{\tau_{q}}^{n}, \quad \text { a.s. }
$$

Enlarging $E^{\prime}$ by a countable family of $P$-null sets (one for each $\tau_{q}$ ), we obtain a set $E$ of $P$-measure zero (belonging to $\mathcal{F}_{0}$ due to the "usual" condition) outside of which (2) holds.

In order to prove (3) let us observe that we can write

$$
\tilde{A}_{\tau}^{n}=\sum_{k=1}^{k_{n}} A_{t_{k}^{n}}^{n} I\left(t_{k-1}^{n}<\tau \leqslant t_{k}^{n}\right) .
$$

Since $\tau$ is a stopping time, the event $\left\{t_{k-1}^{n}<\tau \leqslant t_{k}^{n}\right\}$ belongs to $\mathcal{F}_{t_{k}^{n}}$. If we define

$$
\rho^{n}(\tau)=0 \quad \text { if } \quad \tau=0, \quad \rho^{n}(\tau)=t_{k}^{n} \quad \text { if } \quad t_{k-1}^{n}<\tau \leqslant t_{k}^{n},
$$

then $\rho^{n}(\tau)$ is a stopping time with respect to the discrete filtration $\left\{\mathcal{F}_{t}\right\}_{t \in \theta_{n}}$, $\rho^{n}(\tau) \geqslant \tau, \rho^{n}(\tau) \searrow \tau$ and

$$
\tilde{A}_{\tau}^{n}=A_{\rho^{n}(\tau)}^{n} .
$$

By the properties of the (discrete) Doob-Meyer decomposition

$$
E A_{\rho^{n}(\tau)}^{n}=E X_{\rho^{n}(\tau)}^{n}=E X_{\rho^{n}(\tau)} \searrow E X_{\tau}=E A_{\tau}
$$

We have proved that $A$ is predictable. The proof of its uniqueness is standard (see e.g. Lemma 22.11 and Proposition 15.2 in [4]) and does not involve advanced tools.

\section{References}

1. Aldous, D.J. (1985): Exchangeablility and related topics. In: Aldous, D.J, Ibragimov, I.A., Jacod, J.,École d'été de probabilités de Saint-Flour, XIII - 1983, 1-198, Lecture Notes in Math., 1117, Springer Berlin Heidelberg

2. Dellacherie, C., Doléans-Dade, C. (1971): Un contre-exemple au problème des laplaciens approchés. In: Séminaire Probab. V, 127-137, Lecture Notes in Math., 191, Springer Berlin Heidelberg

3. Doléans, C. (1967): Processus croissants naturels et processus croissants très bien mesurables, C. R. Acad. Sci. Paris Sér. A-B, 264, 874-876

4. Kallenberg, O. (1997): Foundations of Modern Probability, Springer New York Berlin Heidelberg 
5. Komlós, J. (1967): A generalization of a problem of Steinhaus, Acta Math. Acad. Sci. Hungar., 18, 217-229

6. Meyer, P.A. (1963): Decomposition of supermartingales: The uniqueness theorem, Illinois J. Math., 7, 1-17

7. Meyer, P.A. (1976): Un cours sur les intégrales stochastiques. In: Séminaire Probab. X, 127-137, Lecture Notes in Math., 511, Springer Berlin Heidelberg

8. Rao, K.M. (1969): On decomposition theorems of Meyer, Math. Scand., 24, 66-78 